\title{
Tumour infiltrating lymphocytes correlate with improved survival in patients with oesophageal adenocarcinoma
}

\author{
Fergus Noble ${ }^{1,3} \cdot$ Toby Mellows $^{1} \cdot$ Leo H. McCormick Matthews ${ }^{1}$. \\ Adrian C. Bateman ${ }^{2} \cdot$ Scott Harris $^{5} \cdot$ Timothy J. Underwood $^{1,3} \cdot$ James P. Byrne $^{3}$. \\ Ian S. Bailey ${ }^{3}$ - Donna M. Sharland ${ }^{3} \cdot$ Jamie J. Kelly $^{3} \cdot$ John N. Primrose ${ }^{1,3}$. \\ Surinder S. Sahota ${ }^{1}$ Andrew R. Bateman $^{1,4}$ - Gareth J. Thomas ${ }^{1,2}$. \\ Christian H. Ottensmeier ${ }^{1,4}$
}

Received: 12 June 2015 / Accepted: 8 March 2016 / Published online: 28 March 2016 (C) The Author(s) 2016. This article is published with open access at Springerlink.com

\begin{abstract}
Background Oesophageal adenocarcinoma (OAC) is increasingly common in the west, and survival remains poor at $10-15 \%$ at 5 years. Immune responses are increasingly implicated as a determining factor of tumour progression. The ability of lymphocytes to recognise tumour antigens provides a mechanism for a host immune attack against cancer providing a potential treatment strategy. Materials and Methods Tumour infiltrating lymphocytes (TILs: CD3+, CD4+, CD8+ and FOXp3+) were assessed
\end{abstract}

Presented in part (orally) at the International Society for Diseases of the Esophagus annual scientific meeting held in Vancouver, Canada, 2014, and the Association of Upper Gastrointestinal Surgeons of Great Britain and Ireland meeting held in Brighton, England, 2014. The abstracts were published in abstract books: Noble F, Mellows T, McCormick Matthews L, Bateman AC, Byrne JP, Bailey IS, Sharland D, Kelly JJ, Underwood TJ, Primrose JN, Sahota SS, Bateman AR, Thomas GJ, Ottensmeier CH (2014) Tumour Infiltrating Lymphocytes Associate with Improved Survival in Oesophageal Adenocarcinoma. Br J Surg 101 (S6): 1-54.

Noble F, Mellows T, McCormick Matthews L, Bateman AC, Byrne JP, Bailey IS, Sharland D, Kelly JJ, Underwood TJ, Primrose JN, Sahota SS, Bateman AR, Thomas GJ, Ottensmeier CH (2014) Tumour Infiltrating Lymphocytes Associate with Improved Survival in Oesophageal Adenocarcinoma. Dis Esophagus 27 (S1): 3A-176A.

Electronic supplementary material The online version of this article (doi:10.1007/s00262-016-1826-5) contains supplementary material, which is available to authorized users.

Fergus Noble

f.noble@soton.ac.uk

Cancer Sciences Unit, Faculty of Medicine, University of Southampton, Somers Cancer Research Building (MP824), Southampton General Hospital, Tremona Road, Southampton, UK by immunohistochemistry using tissue microarrays in a contemporary and homogeneous cohort of OAC patients $(n=128)$ undergoing curative treatment.

Results Multivariate analysis identified three independent prognostic factors for improved cancer-specific survival (CSS): increased CD8+ TILs $(p=0.003)$, completeness of resection $(p<0.0001)$ and lower pathological $\mathrm{N}$ stage $(p<0.0001)$. Independent prognostic factors for favourable disease-free survival included surgery-only treatment $(p=0.015)$, completeness of resection $(p=0.001)$, increased CD8+ TILs $(p<0.0001)$ and reduced pathological N stage $(p<0.0001)$. Higher levels of TILs in the pathological specimen were associated with significant pathological response to neoadjuvant chemotherapy (NAC). On multivariate analysis increased levels of CD4+ $(p=0.017)$ and CD8+ TILs $(p=0.005)$ were associated with significant local tumour regression and lymph node downstaging, respectively.

Discussion Our results establish an association of TILs and survival in OAC, as seen in other solid tumours, and identify particular TIL subsets that are present at higher levels in patients who responded to NAC compared to non-responders. These findings highlight potential therapeutic strategies in EAC based on utilising the host immunological response and highlight the immune responses biomarker potential.

2 Department of Cellular Pathology, University Hospital Southampton NHS Foundation Trust, Southampton, UK

3 Department of Surgery, University Hospital Southampton NHS Foundation Trust, Southampton, UK

4 Cancer Care, University Hospital Southampton NHS Foundation Trust, Southampton, UK 
Keywords Cytotoxic T lymphocyte - Immune response · Immunotherapy · Oesophageal adenocarcinoma .

Regulatory $\mathrm{T}$ cells $\cdot$ Tumour regression

\begin{tabular}{|c|c|}
\hline \multicolumn{2}{|c|}{ Abbreviations } \\
\hline ASA & $\begin{array}{l}\text { American Society of Anesthesiologists physical } \\
\text { status classification system }\end{array}$ \\
\hline $\mathrm{CD}$ & Cluster of differentiation \\
\hline CSS & Cancer-specific survival \\
\hline DFS & Disease-free survival \\
\hline FOXp3 & Forkhead box p3 \\
\hline $\mathrm{LN}$ & Lymph node \\
\hline NAC & Neoadjuvant chemotherapy \\
\hline NLR & Neutrophil/lymphocyte ratio \\
\hline OAC & Oesophageal adenocarcinoma \\
\hline OSCC & Oesophageal squamous cell carcinoma \\
\hline PARP & Poly-ADP ribose polymerase inhibitors \\
\hline TIL & Tumour infiltrating lymphocyte \\
\hline TMA & Tissue microarray \\
\hline TNM & The TNM classification of malignant tumours \\
\hline TRG & Tumour regression grade \\
\hline WCC & White cell count \\
\hline
\end{tabular}

\section{Introduction}

Virchow first described the association of lymphocyte infiltration with solid tumours in 1863 [1]. Immune responses against malignant cells, whether systemic or within the tumour microenvironment, are increasingly implicated as a determining factor in tumour progression [2]. The most comprehensive studies, to establish this hallmark of cancer [3], have been in colorectal tumours [4]. Specific tumour infiltrating lymphocyte (TIL) subsets communicate and function to affect tumour growth, and the balance of these effects leads to either tumour regression or tumour promotion. An anti-tumour effect is mediated by the combination of cancer cell lysis and the production of cytotoxic cytokines, supported by cluster of differentiation (CD)4+ $\mathrm{T}$ helper cells. In contrast, $\mathrm{T}$ regulatory cells, Forkhead box $\mathrm{P} 3+(\mathrm{FOXp} 3+) \mathrm{T}$ cells, act to suppress the immune response of other cells and so affect the inflammatory process indirectly [2]. Therefore, understanding the impact of specific subsets of immune cells that infiltrate tumours is important for making rational decisions in the development of targeted therapies.

Oesophageal adenocarcinoma (OAC) is becoming increasingly common in the Western world, and despite a variety of strategies to improve outcome [5], survival

5 Public Health Sciences and Medical Statistics, Faculty of Medicine, University of Southampton, Southampton, UK remains poor at $10-15 \%$ at 5 years [6]. Radical treatment with curative intent includes neoadjuvant therapy and oesophagogastrectomy. Neoadjuvant chemotherapy (NAC) delivers improved survival in a small percentage of patients, in whose tumours there is a significant pathological response to treatment [7]. However, approximately twothird of patients present with advanced, incurable disease at diagnosis. Biological therapies such as growth factor blockers, poly-ADP ribose polymerase (PARP) inhibitors, vaccines and monoclonal antibodies are being evaluated and are yet to enter routine clinical practice [8]. The rapid rise in incidence of OAC in the west means that novel therapies are urgently required, either as single agents or for use in combination with conventional treatments.

In particular, the ability of CD8+ lymphocytes to recognise tumour antigens has been well documented [9], and tumour regression is observed when tumour-reactive $\mathrm{T}$ lymphocytes invade cancers [10]. Before considering immune therapies as a potential treatment in OAC, it is important to understand whether immune cells are present in OAC, in which subsets of cells are observed and whether or not they have prognostic significance.

The effects of TILs have been studied in a range of solid tumours, and high TIL density correlates with better survival (reviewed in [11]). A few initial studies have examined the association of TILs in OAC with prognosis $[12,13]$. These studies have provided conflicting results, and no study has assessed TIL density in resected tumours after NAC and whether or not there is a link to outcome. Schumacher et al. observed intratumoural CD8+ $\mathrm{T}$ cell infiltration to correlate with improved survival in a small heterogeneous cohort of OAC $(n=37)$ and oesophageal squamous cell carcinoma (OSCC) $(n=33)$ patients [14]. Additional studies have shown that the overall grade of TIL density at the invasive margin correlates with improved survival in patients with gastroesophageal cancer [12]. In contrast, Zingg et al. did not find any independent associations between differing TIL subsets and survival in OAC patients who received multimodal therapy with either neoadjuvant chemoradiotherapy or surgery alone. However, they did demonstrate in univariate analyses that particular subtypes of TIL conferred a better survival when dichotomised at median counts $(\mathrm{CD} 3+, \mathrm{CD} 8+, \mathrm{FOXp} 3+, \mathrm{CD} 8+: \mathrm{CD} 4+)$ [15]. Therefore, the prognostic value of TILs in OAC remains to be established and the composition of TIL density following NAC in OAC is yet to be assessed.

In this study, we assessed the immune infiltrate in a large contemporary cohort of OAC $(n>120)$ to identify association with survival and clinicopathological disease characteristics. Specifically, we examined TILs, as their presence would suggest that active immunotherapy might be attractive in this condition. A link between pre-existing immunity might then also allow us to stratify patients into 
groups, more or less likely to benefit from immunomodulation such as checkpoint blockade, which allows a release of pre-existing immune responses for clinical benefit. In addition, we analyse TIL frequencies following NAC and assess the relationship between TILs and disease outcome.

\section{Materials and methods}

\section{Patient selection}

A prospectively collected database of consecutive patients undergoing oesophagogastric resection for OAC treated at University Hospital Southampton NHS Foundation Trust between January 2005 and December 2010 was reviewed. Patients were excluded from the study if they had achieved a complete pathological response (tumour regression grade (TRG) 1; no residual tumour), if they died post-operatively as an inpatient, or if no histopathological tissue was available. Ethical approval was received from the Southampton and South West Hampshire Research and Ethics Committee (REC 09/H0504/66) and approved by the local research and development department (RHM CAN0649).

\section{Patient clinical, pathological, treatment and follow-up characteristics}

All patients $(n=128$, Table 1$)$ were discussed at a specialist multidisciplinary team meeting. Standard staging investigations included endoscopic ultrasonography, high-resolution computed tomography, integrated fluorodeoxyglucose positron emission tomography/computed tomography and staging laparoscopy, where indicated and were uniformly applied during the study interval. Patients considered suitable for potential surgical resection with tumours staged as T2 N0 M0 or above were considered for NAC.

NAC consisted of three 21-day cycles of anthracycline, platinum and fluoropyrimidine: ECF (epirubicin $50 \mathrm{mg} / \mathrm{m}^{2}$, cisplatin $60 \mathrm{mg} / \mathrm{m} 2$, both intravenously on day 1 and venous infusion 5-FU $200 \mathrm{mg} / \mathrm{m} 2$ per day for 21 days), ECX (epirubicin $50 \mathrm{mg} / \mathrm{m} 2$, cisplatin $60 \mathrm{mg} / \mathrm{m} 2$, both intravenously on day 1 and capecitabine $625 \mathrm{mg} / \mathrm{m} 2$ orally twice daily for 21 days) or EOX (epirubicin $50 \mathrm{mg} / \mathrm{m} 2$ i.v. bolus and oxaliplatin $130 \mathrm{mg} / \mathrm{m} 2$ i.v. infusion over $2 \mathrm{~h}$ on day 1 , capecitabine $625 \mathrm{mg} / \mathrm{m} 2$ orally twice daily for 21 days).

Pathological status after chemotherapy was assessed using the TRG system developed by Mandard et al. [16] who scored regression based on the degree of fibrosis and number of residual cancer cells (TRG 1-5). TRG was scored by specialist gastrointestinal pathologists, initially by one pathologist (Adrian C Bateman) prior to its introduction by all pathologists as part of routine pathological reporting. All dissected lymph nodes were stained with
Table 1 Clinicopathological characteristics of patient cohort for which formed TMAs

\begin{tabular}{|c|c|}
\hline & $n=128$ \\
\hline Operation age* & $67.77(45.48-85.41)$ \\
\hline Sex ratio $(M: F)$ & $112: 16$ \\
\hline \multicolumn{2}{|l|}{ ASA } \\
\hline 1 & $13(10.2)$ \\
\hline 2 & $93(72.4)$ \\
\hline 3 & $22(17.3)$ \\
\hline \multicolumn{2}{|l|}{ Tumour site } \\
\hline Lower $1 / 3$ & $46(35.9)$ \\
\hline OGJ-S1 & $25(19.5)$ \\
\hline OGJ-S2 & $26(20.3)$ \\
\hline OGJ-S3 & $31(24.2)$ \\
\hline \multicolumn{2}{|l|}{ Type } \\
\hline $\mathrm{AC}$ & $128(100)$ \\
\hline \multicolumn{2}{|l|}{ pT or ypT } \\
\hline $\mathrm{T} 1$ & $29(22.7)$ \\
\hline $\mathrm{T} 2$ & $30(23.4)$ \\
\hline $\mathrm{T} 3$ & $65(50.8)$ \\
\hline $\mathrm{T} 4$ & $4(3.1)$ \\
\hline \multicolumn{2}{|l|}{$\mathrm{pN}$ or $\mathrm{ypN}$} \\
\hline No & $59(46.1)$ \\
\hline N1 & $28(21.9)$ \\
\hline N2 & $21(16.4)$ \\
\hline N3 & $20(15.6)$ \\
\hline \multicolumn{2}{|l|}{$\mathrm{pM}$ or $\mathrm{ypM}$} \\
\hline M0 & $125(97.7)$ \\
\hline M1 & $3(2.3)$ \\
\hline Resection clearance (R0) & $104(81.3)$ \\
\hline Vascular invasion & $51(39.8)$ \\
\hline Lymphatic invasion & $21(16.4)$ \\
\hline Perineural invasion & $17(13.3)$ \\
\hline Neoadjuvant chemotherapy & $76(59.4)$ \\
\hline \multicolumn{2}{|l|}{ Neoadjuvant regime } \\
\hline ECX & $64(50)$ \\
\hline EOX & $10(7.8)$ \\
\hline $\mathrm{ECF}$ & $2(1.6)$ \\
\hline \multicolumn{2}{|l|}{ Tumour regression grade } \\
\hline 1 & $0(0)$ \\
\hline 2 & $11(8.6)$ \\
\hline 3 & $10(7.8)$ \\
\hline 4 & $29(22.7)$ \\
\hline 5 & $26(20.3)$ \\
\hline Not assessed & $0(0)$ \\
\hline Surgery only & $52(40.6)$ \\
\hline Nodal downstaging & $30 / 76(39.5)$ \\
\hline
\end{tabular}

Values in parentheses are percentages unless indicated

ASA American Society of Anesthesiologists physical status classification system

* Values in parentheses are range 
haematoxylin and eosin and microscopically analysed for metastatic disease. Lymph node downstaging was defined by lymph nodes being positive for signs of cancer $(\mathrm{cN}+)$ at diagnosis, assessed radiologically (computed tomography, positron emission tomography, endoscopic ultrasonography) and then pathologically recorded as the lymph nodes showing no signs of cancer spread (ypN0) after NAC given prior to surgery as previously described [17].

Surgery was performed after initial staging or 4-6 weeks following NAC as previously described [18].

Data recorded included demographics, tumour characteristics, resection type and histopathological analysis of the surgical specimen. The TNM classification of malignant tumours (TNM) 7 was used to report tumour stage after analysis of pathology reports [19]. Pathological tumour clearance ("R"-status) was determined according to the Royal College of Pathologists' guidance [20].

All patients were cared for by a specialist oesophagogastric team as previously described [18]. Recurrence of disease during follow-up was defined as the first site or sites of recurrence with radiological or pathological confirmation. Cancerspecific survival (CSS) was defined as time of operation to death in the absence of other causes of death. For assessment of disease-free survival (DFS), recurrence was defined as time from operation to development of local, nodal (regional) and distant metastasis (whichever occurred first).

\section{Immunohistochemistry}

Post-operative tumour histology was reviewed by pathologists (Gareth J. Thomas and Adrian C. Bateman) blinded to treatment and outcome, and a suitable paraffin block was selected. Tissue microarrays (TMAs) were constructed using triplicate, randomly selected, paraffin-embedded 1-mm tumour cores (Aphelys Minicore 2, Mitogen, Harpenden, UK). To assess the immune infiltrate, $4-\mu \mathrm{m}$ sections of TMA blocks were used. Automated immunostaining (Ventana XT, Ventana, Tucson, AZ, USA) was performed in a clinical pathology accredited cellular pathology department using antibodies optimised to national diagnostic standards (National External Quality Assessment Service). Antibodies to assess antigens were as follows: CD3, pan T cells; CD4, helper T cells; CD8, cytotoxic $\mathrm{T}$ cells (all from Novocastra, Milton Keynes, UK); and FOXp3, regulatory T cells (eBioscience, Hatfield, UK). Patients $(n=13)$ with complete pathological response (TRG 1) were excluded due to the inability to assess TILs due to the lack of tumour.

\section{Immunohistochemical evaluation}

All sections were digitally image captured to enable sections to be scored under the supervision of an experienced pathologist (Gareth J. Thomas). Three high-powered fields ( $\times 400$ magnification) with the highest density of the marker of interest were scored, with a mean taken, providing a total scoring area of $0.1944 \mathrm{~mm} 2$. This is in keeping with previous studies assessing intratumoural immune infiltration [21].

Training of the observers was undertaken on a series of OACs from the TMA. A total of 25 randomly selected patients from the total cohort were used to assess the intraobserver and interobserver variation in the TIL scoring. Two observers (Fergus Noble and Leo M. Matthews) assessed the TIL infiltrate independently and without knowledge of clinicopathological information. The interobservers' intraclass correlation coefficient was $>0.7$ and was considered acceptable in keeping with previous studies [22]. Fergus Noble scored all slides, and these data were used in the analysis [23].

Following scoring, TIL density data were grouped to facilitate some of the statistical analysis. This was established using justifiable cut-offs (high-low/either side of the median).

\section{Preoperative systemic inflammatory and nutritional blood-borne markers analysed}

Preoperative blood samples were taken for routine laboratory analysis of full blood count (FBC) and albumin in the preoperative period (within 1 week of resection). The white cell count (WCC) (reference range 4.0-11.0 × 109/1), platelet count (reference range 150-400 $\times 109 / 1$ ), neutrophil count (reference range $2.0-7.5 \times 109 / 1)$ and lymphocyte count (reference range $1.5-4.0 \times 109 / 1$ ) were analysed with an automated haematological blood analyser (Sysmex TS-500 (Sysmex UK Ltd)). Serum concentrations of albumin (normal range: $35-48 \mathrm{~g} / \mathrm{l}$ ) were measured in an autoanalyser (UniCel DxC800 (Beckmann Coulter Inc)). The coefficient of variation for these methods, over the range of measurement, was less than $2 \%$ as established by routine quality control. All patients were free from infection at the time of blood collection as determined by clinical assessment.

The neutrophil/lymphocyte ratio (NLR) was calculated by dividing the absolute neutrophil count by the absolute lymphocyte count (reference range 0.5-5) and represents an inexpensive measure of systemic inflammation [24].

\section{Statistical analysis}

Descriptive data are represented as median and range unless indicated. The Kruskal-Wallis, Mann-Whitney U and Pearson's Chi-squared tests were used as appropriate for comparisons of groups. Kaplan-Meier, univariate and multivariate Cox logistic regression modelling were 
used to assess the relationship between immune infiltration with CSS, DFS and response to NAC as outcomes. All factors that showed statistical significance on univariate analysis were entered to derive the final model using the backward stepwise likelihood ratio method. CSS and DFS curves of the patients were plotted by using the KaplanMeier method and analysed using the log-rank test. Stratified analyses were performed based on receipt of NAC and pathological response to chemotherapy. A $p$ value $<0.05$ was considered statistically significant for all tests. Statistical analysis was performed with statistical package for the social sciences version 21 (SPSS $®$, Chicago, Illinois, USA).

\section{Results}

\section{Study patients}

A total of 128 patients were included in the study with a median follow-up of 3.5 years (95\% CI 2.629-4.342). The study population had a median age at operation of 68 years (range, 46-85 years) and was predominately male (88\%). The majority had pathologically advanced staged, pT/ ypT3 (51\%) and node-positive disease (54\%), which were located either at the oesophagogastric junction (64\%) or in the lower oesophagus (36\%).

In total, $76(59.4 \%)$ patients received multimodal therapy, consisting of NAC and surgery, with 52 (40.6\%) patients proceeding directly to surgery alone. Of the patients who received multimodal therapy, 11 (15\%) patients had a significant local tumoural response (TRG $2)$, and $30(40 \%)$ patients had a significant lymph node response (lymph node downstaging: $\mathrm{cN}+$ to $\mathrm{ypN} 0$ ) to NAC.

Detailed patient characteristics and clinical and pathological outcomes are summarised in Table 1.

\section{The relationship of intratumoural infiltrating lymphocytes and clinicopathological characteristics}

Representative TIL staining is shown in supplemental Figure S1. The relationship of patient and tumour characteristics to TILs is presented in supplemental Table S1 and supplemental Table S2, respectively.

A lower TIL density, across all subsets, was significantly associated with increasing stage of disease ( $\mathrm{T}$ and $\mathrm{N}$ stage) and with vascular, lymphatic and perineural invasion (supplemental Table S2).

Higher TIL counts were observed in female compared to male patients (supplemental Table S1). This was statistically significant for CD4+ $(p=0.036), \mathrm{CD} 8+(p=0.037)$ and FOXp3 $3=0.045)$ cells. No statistically significant



Fig. 1 Frequency distribution of CD8+ TILs by treatment modality highlighting the low proportion of patients with high TIL infiltration

differences were found regarding premorbid status (performance status, ASA grade, age or smoking status, supplemental Table S1).

The relationship of TILs and outcome was not statistically different between patients treated with multimodal therapy, NAC and surgery, or surgery alone and is presented in supplemental Table S1 and supplemental Table S3. The distribution of TILs was positively skewed with a heavier left than right tail indicating a low proportion of patients have high levels of infiltration with lymphocytes. The distribution of CD8+ TILs is shown as an example in Fig. 1.

The number of TILs positively correlated with each other for all subtypes (range of correlation coefficient $=0.677-0.905 ; p<0.001)$ and is detailed in supplemental Table S4.

\section{Prognostic significance of intratumoural infiltrating lymphocytes}

Median follow-up was 3.5 years with the results of the Cox proportional hazard model for predictors of CSS and DFS shown in Table 2 and supplemental Table S5, respectively. Multivariate analysis identified independent prognostic factors for improved CSS to be reduced pathological $\mathrm{N}$ stage $(p<0.0001)$, higher CD8+ TILs $(p=0.003)$ and completeness of resection $(p<0.0001)$. The hazard ratio for higher number of CD8+ TILs was $0.847(95 \%$ CI 0.760-0.944). Segregation of the TIL data either at 
Table 2 Univariate and multivariate Cox regression analyses of patient and tumour factors with OAC-specific survival




Table 2 continued

\begin{tabular}{|c|c|c|c|c|c|c|}
\hline & \multicolumn{3}{|c|}{ Univariate } & \multicolumn{3}{|c|}{ Multivariate } \\
\hline & HR & $95 \% \mathrm{CI}$ & $p$ value & HR & $95 \% \mathrm{CI}$ & $p$ value \\
\hline R0 & 1 & Ref & & 1 & Ref & \\
\hline $\mathrm{R} 1$ & 3.027 & $1.737-5.273$ & $<0.0001$ & 3.134 & $1.699-5.783$ & $<0.0001$ \\
\hline
\end{tabular}

Bold values indicate variables of significance

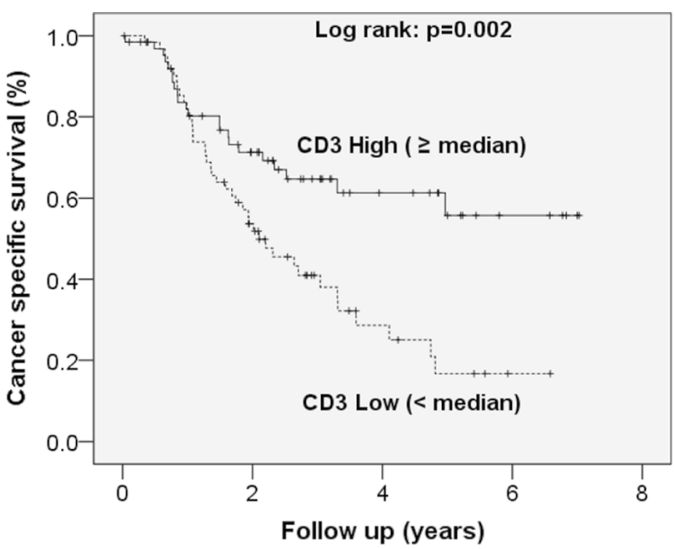

No. at

\begin{tabular}{lllll} 
risk & & & & \\
\hline Years & 0 & 2 & 4 & 6 \\
CD3 & 65 & 37 & 15 & 5 \\
High & & & & \\
CD3 & 63 & 29 & 8 & 1 \\
Low & & & &
\end{tabular}

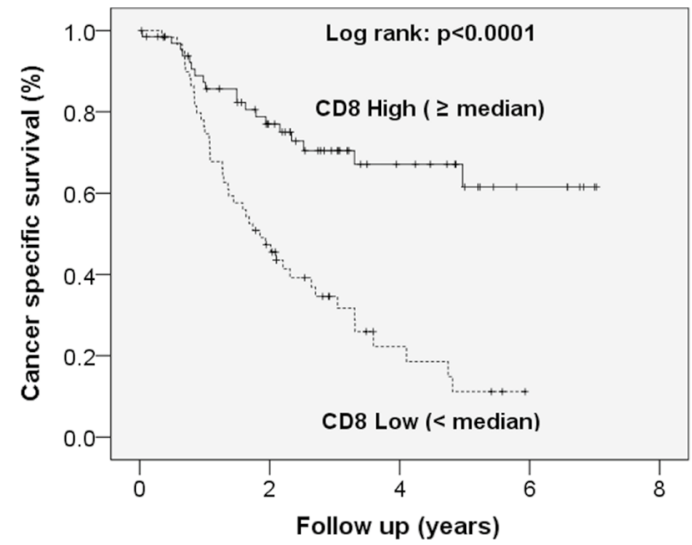

No. at

risk

$\begin{array}{lllll}\text { Years } & 0 & 2 & 4 & 6 \\ \text { CD8 } & 67 & 40 & 17 & 6 \\ \text { High } & & & & \\ \text { CD8 } & 61 & 26 & 6 & 0 \\ \text { Low } & & & & \end{array}$

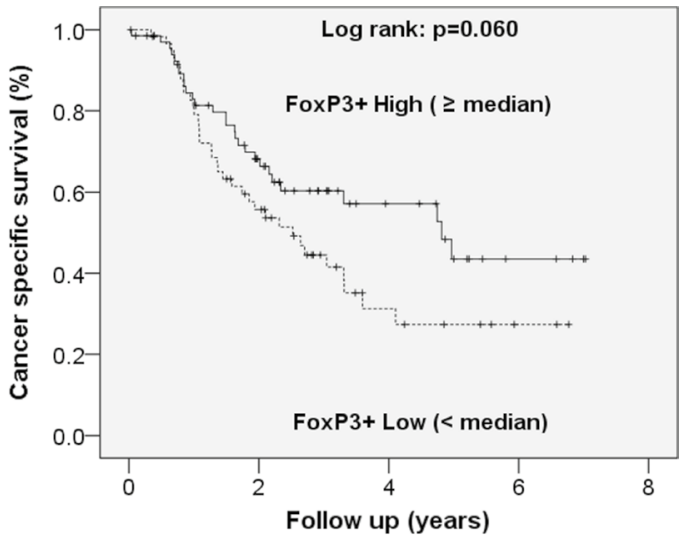

No. at

risk

$\begin{array}{lllll}\text { Years } & 0 & 2 & 4 & 6 \\ \begin{array}{l}\text { Foxp3+ } \\ \text { High }\end{array} & 68 & 37 & 15 & 4 \\ \begin{array}{l}\text { Foxp3+ } \\ \text { Low }\end{array} & 60 & 29 & 8 & 2\end{array}$

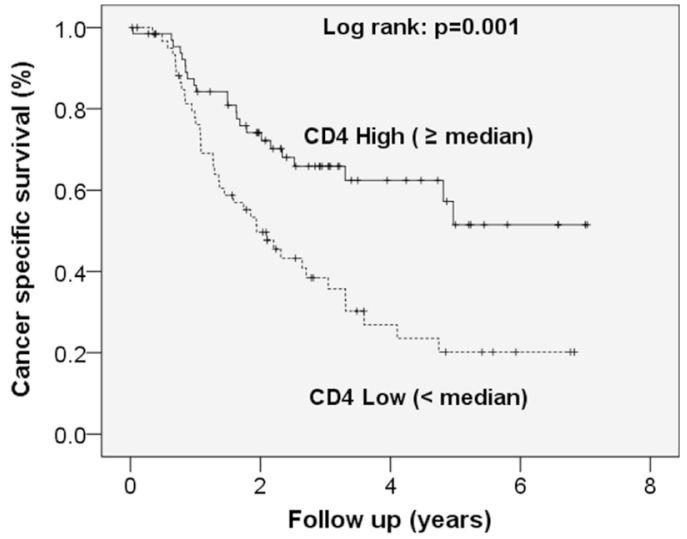

No. at

risk

$\begin{array}{lllll}\text { Years } & 0 & 2 & 4 & 6 \\ \text { CD4 } & 66 & 39 & 15 & 4 \\ \text { High } & & & & \\ \text { CD4 } & 62 & 27 & 8 & 2 \\ \text { Low } & & & & \end{array}$

Fig. 2 Kaplan-Meier curves of cancer-associated survival revealing prognostic significance of TILs in oesophageal adenocarcinoma 
Table 3 Clinical and pathological factors in OAC patients that received chemotherapy $(n=76)$ based on their response to neoadjuvant chemotherapy ( $\mathrm{LN}$ downstaged)

\begin{tabular}{|c|c|c|c|}
\hline & Responder $n=30$ & $\begin{array}{l}\text { Non-responder } \\
n=46\end{array}$ & $p$ value \\
\hline Age* & $\begin{array}{l}63.68(45.48- \\
77.75)\end{array}$ & $\begin{array}{l}62.62(50.67- \\
81.28)\end{array}$ & 0.941 \\
\hline \multicolumn{4}{|l|}{ Sex } \\
\hline Male & $29(96.7)$ & $39(84.8)$ & \multirow[t]{2}{*}{0.101} \\
\hline Female & $1(3.3)$ & $7(15.2)$ & \\
\hline \multicolumn{4}{|c|}{ Performance status } \\
\hline 0 & $8(26.7)$ & $14(30.4)$ & \multirow[t]{3}{*}{0.446} \\
\hline 1 & $20(66.7)$ & $32(69.6)$ & \\
\hline 2 & $2(6.7)$ & $0(0)$ & \\
\hline \multicolumn{4}{|l|}{ ASA } \\
\hline 1 & $5(16.7)$ & $5(11.1)$ & \multirow[t]{3}{*}{0.859} \\
\hline 2 & $20(66.7)$ & $37(80.0)$ & \\
\hline 3 & $5(16.7)$ & $4(8.9)$ & \\
\hline \multicolumn{4}{|l|}{ урт } \\
\hline 1 & $11(36.7)$ & $2(4.3)$ & \multirow[t]{4}{*}{$<0.0001$} \\
\hline 2 & $10(33.3)$ & $11(23.9)$ & \\
\hline 3 & $9(30.0)$ & $31(67.4$ & \\
\hline 4 & $0(0)$ & $2(4.3)$ & \\
\hline \multicolumn{4}{|l|}{ ypN } \\
\hline 0 & $30(100)$ & $3(6.5)$ & \multirow[t]{4}{*}{$<0.0001$} \\
\hline 1 & $0(0)$ & $15(32.6)$ & \\
\hline 2 & $0(0)$ & $15(32.6)$ & \\
\hline 3 & $0(0)$ & $13(28.3)$ & \\
\hline \multicolumn{4}{|c|}{ Tumour response } \\
\hline Yes (TRG2) & $11(36.7)$ & $0(0)$ & \multirow[t]{2}{*}{$<0.0001$} \\
\hline $\begin{array}{l}\text { No (TRG } \\
3-5)\end{array}$ & $19(63.3)$ & $46(100)$ & \\
\hline \multicolumn{4}{|l|}{ Differentiation } \\
\hline G1—well & $5(16.7)$ & $37(80.4)$ & \multirow[t]{3}{*}{0.036} \\
\hline $\begin{array}{l}\text { G2-Moder- } \\
\text { ate }\end{array}$ & $9(30.0)$ & $1(2.2)$ & \\
\hline G3-poor & $16(53.3)$ & $11(23.9)$ & \\
\hline \multicolumn{4}{|c|}{ Resection clearance } \\
\hline R0 & $2(6.7)$ & $12(26.1)$ & \multirow[t]{2}{*}{0.034} \\
\hline $\mathrm{R} 1$ & $28(93.3)$ & $34(73.9)$ & \\
\hline \multicolumn{4}{|c|}{ Immunohistochemistry values* } \\
\hline CD3 & $15.15(0.00-44.30)$ & $11.20(0.00-64.00)$ & 0.007 \\
\hline CD4 & $5.00(0.00-27.00)$ & $2.30(0.00-20.00)$ & 0.025 \\
\hline CD8 & $8.65(0.00-20.70)$ & $4.50(0.00-18.70)$ & 0.002 \\
\hline FOXp3+ & $1.85(0.00-11.30)$ & $1.00(0.00-9.30)$ & 0.074 \\
\hline
\end{tabular}

Values in parentheses are percentages unless indicated

* Values in parentheses are range

Bold values indicate variables of significance

the medians (low $<$ median or high $\geq$ median TIL levels) allowed Kaplan-Meier survival analysis; the data presented in Fig. 2 demonstrate that higher numbers were associated with improved CSS. CD8+ TILs $\geq 5$ were associated with better CSS [CD8+ TILs $\geq 5$ : mean (median not yet reached) CSS 5.1 years, $95 \%$ CI 4.4-5.8, vs. CD8+ TILs < 5: median CSS 1.9 years, $95 \%$ CI 1.3-2.4, $p<0.0001$ ].

Multivariate analysis identified independent prognostic factors for reduced DFS as lower CD8+ TIL numbers $(p<0.0001)$, pathological $\mathrm{N}$ stage $(p<0.0001)$, incompleteness of resection $(p=0.001)$ and multimodal treatment $(p=0.015)$. The hazard ratio for higher number of CD8+ TILs was 0.894 (95 \% CI 0.844-0.948). KaplanMeier survival analysis is presented in supplemental Figure S2 showing that higher levels of TILs were associated with improved DFS. CD8+ TILs $\geq 5$ were associated with better DFS (CD8+ TILs $\geq 5$ : mean (median not yet reached) DFS 4.7 years, $95 \%$ CI 3.9-5.4, vs. CD8+ TILs < 5: median DFS 1.2 years, $95 \%$ CI $0.9-1.5, p<0.0001$ ).

\section{The relationship of intratumoural infiltrating lymphocytes and response to NAC}

Significantly higher TIL numbers were found in the tumours of patients who had a better pathological response to NAC, both when assessing response based on the tumour (TRG) and the lymph nodes (downstaging of lymph nodes from pretreatment clinical staging to pathological $\mathrm{N}$ stage), as detailed in Tables 3, 4, supplemental Table S6, supplemental Table S7 and supplemental Figure S3. There was a higher $\mathrm{CD} 3+(p=0.007), \mathrm{CD} 4+(p=0.025)$ and CD8+ count $(p=0.002)$ in the tumour of patients whose tumour nodal status was downstaged at pathological evaluation after NAC. Higher TIL frequencies were also seen in patients who had a significant pathological response to NAC (TRG2) compared to those with no significant response (TRG 3-5) (Table 3).

On multivariate analysis higher CD4+ TIL $(p=0.017)$ and CD8+ TIL densities $(p=0.005)$ were associated with significant tumour response (TRG) and lymph node downstaging, respectively, shown in supplemental Table S7 and Table 4.

\section{The relationship of intratumoural infiltrating lymphocytes and preoperative systemic inflammatory markers}

Higher TIL numbers were found in the tumours of patients with normal serum albumin although this did not reach statistical significance [albumin < 35: CD8+ numbers $2.00(0.00-29.30)$ vs. albumin $\geq 35$ : CD8+ numbers 6.00 (0.00-38.00), $p=0.069$ (supplemental Table 1)].

\section{Discussion}

In this study we have analysed the level of TILs in a large homogeneous cohort of oesophagogastric cancer patients 
Table 4 Univariate and multivariate analysis of immunohistochemical markers for response to neoadjuvant chemotherapy (LN downstaged) in OAC

\begin{tabular}{|c|c|c|c|c|c|c|}
\hline & \multicolumn{3}{|c|}{ Univariate } & \multicolumn{3}{|c|}{ Multivariate } \\
\hline & $\mathrm{HR}$ & $95 \% \mathrm{CI}$ & $p$ value & HR & $95 \% \mathrm{CI}$ & $p$ value \\
\hline CD3 & 0.957 & $0.924-0.992$ & 0.015 & & & \\
\hline CD4 & 0.916 & $0.838-1.001$ & 0.052 & & & \\
\hline CD8 & 0.869 & $0.789-0.958$ & 0.005 & 0.869 & $0.789-0.958$ & 0.005 \\
\hline FOXp3+ & 0.868 & $0.734-1.026$ & 0.097 & & & \\
\hline
\end{tabular}

Bold values indicate variables of significance following radical treatment with curative intent. We obtained three major findings: firstly, we have shown associations between pathological stage of disease and TIL density and confirmed the independent association of particular TIL subsets and survival; secondly, we have shown significant correlation between TIL subtypes in OAC, and finally, we found patients with a significantly increased pathological response to NAC had higher levels of TILs in their resected tumour, most notably with CD4+ and CD8+ TILs for local tumour regression and lymph node response, respectively.

Of the TIL subsets analysed, CD8 + T cells had the most significant independent association for both CSS ( $p=0.003$ ) and DFS $(p<0.0001)$. This has been previously suggested to be important in OAC; however, independent association has not been universally verified [12, 14, 15]. Zingg el al. [15] identified on univariate analysis a number of TILs as significant predictive factors; however, these were not independent factors when taking into account the stage of disease. In contrast, Schumacher et al. [14] identified CD8+ TILs to be a significant predictive factor of survival independent of disease stage. The reasons for this discrepancy of findings between studies may relate to the clinicopathological factors used to build the multivariate statistical model, the location of TILs counted and the cut-off values used to dichotomise the TIL frequencies into high and low groups. In our large and homogeneous cohort, we choose to evaluate TIL number as a continuous variable, counted intratumoural TILs and entered a comprehensive number of known clinicopathological predictors of survival into the univariate and subsequent multivariate statistical models. We believe this to be the most robust analysis of the association of TILs with survival on $\mathrm{OAC}$ that has been conducted to date.

In addition to the observed effects TILs have on disease recurrence, this study demonstrates that TILs play a role at different stages of disease. The significant association observed between TILs with $\mathrm{T}$ and $\mathrm{N}$ stage supports the possibility that intratumoural $\mathrm{T}$ cells prevent tumour progression throughout the disease process. These findings are supported by previous smaller studies showing correlation with low TILs and higher stage of disease $[14,15]$.

A novel observation is that the response to chemotherapy links with TIL counts. Previous studies have highlighted increased infiltrate of TILs in OAC tumours after NAC when compared to surgery alone [13]. We did not find any statistically significant difference between TIL levels in those patients who received NAC and those that proceeded directly to surgery as a whole. It would be preferable to evaluate TILs in preoperative biopsies to assess response prior to neoadjuvant therapy. This was attempted but was unsuccessful due to the paucity of tumour material in the small diagnostic endoscopic biopsies unlike the diagnostic biopsies taken for other tumours that tend to be larger, for example breast and colon cancer. However, different and heterogeneous chemotherapy regimens were used in previous studies making comparison difficult. We did find that patients who had a significant response to NAC (as assessed by TRG and $\mathrm{LN}$ response) had a higher level of immune infiltrate. This important finding has been shown in other cancer sites where loss of CD4+ Treg [23] and TIL numbers at the margins of liver metastases [24] predicted for response to chemotherapy [25-27]. The potential immunological mechanisms by which cytotoxic chemotherapy can provide anti-tumour activity are being increasingly highlighted. These include: subverting immunosuppressive mechanisms; exerting stimulatory effects in immune cells; and modulating dying tumour cells, so they regain visibility to the host immune response [28]. The combination of immunological therapies with conventional chemotherapy has been suggested to provide a synergistic effect if the host immunological response is harnessed appropriately [29]. In addition to the effects of chemotherapy on TILs, previous studies have highlighted spontaneous pathological regression of tumours in $13.7 \%(n=17 / 124)$ of OAC cases that have received no NAC when using TRG to assess the resected tumour specimen. This may represent the host immunological response and successful immune attack [30]. Our study would support this hypothesis as significant response to NAC was associated with increased TILs most notably with CD8+ TILs. In other tumour sites, CD8+ infiltration has been shown to correlate with specific immunogenic antigen expression and improved survival [31]. These findings highlight the $\mathrm{T}$ cell effector potential and a means to harness the patient's immune response in cancer.

It is also of interest that increased frequencies of TILs correlate with improved response following the use of 
trastuzumab [32] in light of the ToGA study [33] that may lead to the adjuvant use of trastuzumab in OAC. This adjuvant therapy may lead to a further increase in the adaptive immune response in the tumour as seen in breast cancer [34]. We have previously shown that patients are more likely to respond to chemotherapy if acute phase proteins are normal (serum albumin) by assessing systemic markers of nutrition and the inflammatory response (neutrophil/ lymphocyte ratio, serum albumin) [18]. It is suggested that suboptimal immunological and nutritional status may contribute to tumour development through subversion of tumour immunity $[35,36]$ and this is particularly pertinent to OAC. We found that patients with lower TIL levels were less likely to respond to chemotherapy and had lower serum albumin levels. From our data, it is not possible to know whether high TIL levels lead to a greater responsiveness to chemotherapy or chemotherapy leads to a higher number of TILs in those that respond, and it may well be a combination of the two. The association of poor nutritional status with lower TIL levels and a lack of response are logical and may well be the most likely explanation.

Limitations of this study include its retrospective nature and the associated biases on selection and collection. Patients were excluded if they had a complete pathological response to NAC due to the lack of tumour in the resection specimen and also if there was insufficient material collected. However, this cohort is representative of current clinical practice. We found increasing nodal burden $(p<0.0001)$ to be the best independent prognosticator for worse survival. The cohort was homogenous in terms of staging, histology and treatment algorithms. In addition, patient, tumour factors and survival data are in keeping with published western cohorts making our findings applicable to these populations. The excluded patients with complete pathological response (TRG1) potentially will have had the highest immune response with high TIL levels. This hypothesis is supported by analysis of the cytotoxic response of patients with a complete pathological response in breast cancer patients. Granzyme B and TiA1, cytolytic granules, expressing cells were observed at higher frequency in specimens that had undergone a pathological complete response [25].

Additional criticism could be expressed with regard to the use of TMA cores not being representative of the tumour as a whole. However, multiple studies have utilised this approach and excellent correlation between the two has been established [37]. With respect to scoring TILs, the technique used has been described frequently by other studies with good interobserver and intraobserver correlation. Furthermore, these data were analysed with and without dichotomization so as to limit false-positive results [38, 39].

An unexpected finding in our study was the association of higher TILs for some subsets $(\mathrm{CD} 4+, \mathrm{CD} 8+$ and
FOXp3+) in female patients. This may represent a type 1 error; however, it is well documented that immune cell numbers vary with age and gender. Possible explanations include the inhibitory effect of oestrogen on T-suppressor cells or its stimulatory effect on T helper cells [40]. However, this speculation is outside the remit of this study and will require further analysis. The reason why higher infiltration of regulatory FOXp3+ cells showed favourable prognosis in this study is complex but has been reported in gastric and other tumour types [41, 42]. This warrants further functional analysis, but others have suggested a synergistic role of regulatory cells with other TIL subsets within the tumour [43].

With regard to our novel finding, it remains to be seen whether the composition of TILs can predict for response to chemotherapy prior to treatment and the functional role the immune response plays in improving the response to chemotherapy. Emerging evidence from other solid organ tumours suggests that this might be possible [44]. However, as yet the required sensitivity and specificity have not been met for this to enter clinical practice. A potential clinical application of the TIL response could be in the selection of patients for specific adjuvant therapies, specifically using immunomodulators. In our series, a significant response to chemotherapy (TRG2 or LN downstaging) was associated with high TIL levels, suggesting these patients may benefit from further adjuvant chemotherapy. It would be intriguing in this group in particular to evaluate the long-term outcome after the use of immunomodulators, that release pre-existing anti-tumour $\mathrm{T}$ cells, as has been proposed for aPD1 and aCTLA4 therapies. In contrast, the group of patients who had no or a partial response to chemotherapy (TRG 3-5 or no LN downstaging) with high TIL levels in the pathological specimen additionally may benefit from further adjuvant or alternative chemotherapy in combination with an immunomodulator. In contrast, those patients with low TILs may warrant the use of second-line therapies but it seems less likely that immune attack will be successful, unless second-line therapy can overcome the barriers to immunological visibility of the cancer.

In summary, the results of this study show that local immune responses, in particular the adaptive immune response, are important independent predictors of CSS and DFS in patients with OAC undergoing radical treatment. These findings highlight the role of the adaptive immune response in preventing tumour recurrence in OAC. A generalised immune response was observed with high correlation between TIL subtypes and at all stages of disease. This is pertinent with regard to the design of therapies as it suggests all patients would benefit from treatment that appropriately boosts the immune response. A significant response was associated with higher infiltration with TILs post-therapy. Further work analysing the function of these 
TIL subsets and the correlation of pretreatment TIL densities with response to therapy may unearth predictors of response to chemotherapy in addition to aid design of novel treatments or as a mechanism to improve response to current therapies.

Acknowledgments This study was supported by Cancer Research UK and the Southampton Experimental Cancer Medicine Centre tumour bank.

\section{Compliance with ethical standards}

Conflict of interest The authors declare that they have no conflict of interest.

Open Access This article is distributed under the terms of the Creative Commons Attribution 4.0 International License (http://creativecommons.org/licenses/by/4.0/), which permits unrestricted use, distribution, and reproduction in any medium, provided you give appropriate credit to the original author(s) and the source, provide a link to the Creative Commons license, and indicate if changes were made.

\section{References}

1. Balkwill F, Mantovani A (2001) Inflammation and cancer: back to Virchow? Lancet 357(9255):539-545

2. Grivennikov SI, Greten FR, Karin M (2010) Immunity, inflammation, and cancer. Cell 140(6):883-899

3. Hanahan D, Weinberg RA (2011) Hallmarks of cancer: the next generation. Cell 144(5):646-674

4. Galon J, Costes A, Sanchez-Cabo F, Kirilovsky A, Mlecnik B, Lagorce-Pages C, Tosolini M, Camus M, Berger A, Wind P, Zinzindohoue F, Bruneval P, Cugnenc PH, Trajanoski Z, Fridman WH, Pages F (2006) Type, density, and location of immune cells within human colorectal tumors predict clinical outcome. Science 313(5795): 1960-1964

5. Cowie A, Noble F, Underwood T (2014) Strategies to improve outcomes in esophageal adenocarcinoma. Expert Rev Anticancer Ther 14(6):677-687

6. Lepage C, Rachet B, Jooste V, Faivre J, Coleman MP (2008) Continuing rapid increase in esophageal adenocarcinoma in England and Wales. Am J Gastroenterol 103(11):2694-2699

7. Cunningham D, Allum WH, Stenning SP, Thompson JN, Van de Velde CJ, Nicolson M, Scarffe JH, Lofts FJ, Falk SJ, Iveson TJ, Smith DB, Langley RE, Verma M, Weeden S, Chua YJ, Participants MT (2006) Perioperative chemotherapy versus surgery alone for resectable gastroesophageal cancer. $\mathrm{N}$ Engl $\mathrm{J}$ Med 355(1):11-20

8. Allum WH, Blazeby JM, Griffin SM, Cunningham D, Jankowski JA, Wong R, Association of Upper Gastrointestinal Surgeons of Great B, Ireland tBSoG, the British Association of Surgical O (2011) Guidelines for the management of oesophageal and gastric cancer. Gut 60(11):1449-1472

9. Boon T, Coulie PG, Van den Eynde B (1997) Tumor antigens recognized by $\mathrm{T}$ cells. Immunol Today 18(6):267-268

10. Mukai S, Kjaergaard J, Shu S, Plautz GE (1999) Infiltration of tumors by systemically transferred tumor-reactive T lymphocytes is required for antitumor efficacy. Cancer Res 59(20):5245-5249

11. Gooden MJ, de Bock GH, Leffers N, Daemen T, Nijman HW (2011) The prognostic influence of tumour-infiltrating lymphocytes in cancer: a systematic review with meta-analysis. Br J Cancer 105(1):93-103

12. Dutta S, Crumley AB, Fullarton GM, Horgan PG, McMillan DC (2011) Comparison of the prognostic value of tumour- and patient-related factors in patients undergoing potentially curative resection of oesophageal cancer. World J Surg 35(8):1861-1866

13. Crumley AB, Going JJ, Hilmy M, Dutta S, Tannahill C, McKernan M, Edwards J, Stuart RC, McMillan DC (2011) Interrelationships between tumor proliferative activity, leucocyte and macrophage infiltration, systemic inflammatory response, and survival in patients selected for potentially curative resection for gastroesophageal cancer. Ann Surg Oncol 18(9):2604-2612

14. Schumacher K, Haensch W, Roefzaad C, Schlag PM (2001) Prognostic significance of activated CD8(+) T cell infiltrations within esophageal carcinomas. Cancer Res 61(10):3932-3936

15. Zingg U, Montani M, Frey DM, Dirnhofer S, Esterman AJ, Went P, Oertli D (2010) Tumour-infiltrating lymphocytes and survival in patients with adenocarcinoma of the oesophagus. Eur J Surg Oncol 36(7):670-677

16. Mandard AM, Dalibard F, Mandard JC, Marnay J, Henry-Amar M, Petiot JF, Roussel A, Jacob JH, Segol P, Samama G et al (1994) Pathologic assessment of tumor regression after preoperative chemoradiotherapy of esophageal carcinoma. Clinicopathologic correlations. Cancer 73(11):2680-2686

17. Noble F, Nolan L, Bateman AC, Byrne JP, Kelly JJ, Bailey IS, Sharland DM, Rees CN, Iveson TJ, Underwood TJ, Bateman AR (2013) Refining pathological evaluation of neoadjuvant therapy for adenocarcinoma of the esophagus. World J Gastroenterol 19(48):9282-9293

18. Noble F, Hopkins J, Curtis N, Kelly JJ, Bailey IS, Byrne JP, Bateman AC, Bateman AR, Underwood TJ (2013) The role of systemic inflammatory and nutritional blood-borne markers in predicting response to neoadjuvant chemotherapy and survival in oesophagogastric cancer. Med Oncol 30(3):596

19. Sobin LH, Gospodarowicz MK, Wittekind Ch (eds) (2009) International Union Against Cancer (UICC): TNM classification of malignant tumours, 7th edn. Wiley-Blackwell, Oxford, UK

20. Sagar PM, Johnston D, McMahon MJ, Dixon MF, Quirke $P$ (1993) Significance of circumferential resection margin involvement after oesophagectomy for cancer. Br J Surg 80(11):1386-1388

21. Simpson JA, Al-Attar A, Watson NF, Scholefield JH, Ilyas M, Durrant LG (2010) Intratumoral T cell infiltration, MHC class I and STAT1 as biomarkers of good prognosis in colorectal cancer. Gut 59(7):926-933

22. Klintrup K, Makinen JM, Kauppila S, Vare PO, Melkko J, Tuominen H, Tuppurainen K, Makela J, Karttunen TJ, Makinen MJ (2005) Inflammation and prognosis in colorectal cancer. Eur J Cancer 41(17):2645-2654

23. Jamieson NB, Mohamed M, Oien KA, Foulis AK, Dickson EJ, Imrie CW, Carter CR, McKay CJ, McMillan DC (2012) The relationship between tumor inflammatory cell infiltrate and outcome in patients with pancreatic ductal adenocarcinoma. Ann Surg Oncol 19(11):3581-3590

24. Proctor MJ, Morrison DS, Talwar D, Balmer SM, Fletcher CD, O'Reilly DS, Foulis AK, Horgan PG, McMillan DC (2011) A comparison of inflammation-based prognostic scores in patients with cancer: a glasgow inflammation outcome study. Eur J Cancer 47(17):2633-2641

25. Ladoire S, Arnould L, Apetoh L, Coudert B, Martin F, Chauffert B, Fumoleau P, Ghiringhelli F (2008) Pathologic complete response to neoadjuvant chemotherapy of breast carcinoma is associated with the disappearance of tumor-infiltrating FOXp3+ regulatory T cells. Clin Cancer Res 14(8):2413-2420

26. Halama N, Michel S, Kloor M, Zoernig I, Benner A, Spille A, Pommerencke T, von Knebel DM, Folprecht G, Luber B, Feyen 
N, Martens UM, Beckhove P, Gnjatic S, Schirmacher P, Herpel E, Weitz J, Grabe N, Jaeger D (2011) Localization and density of immune cells in the invasive margin of human colorectal cancer liver metastases are prognostic for response to chemotherapy. Cancer Res 71(17):5670-5677

27. Chan MS, Wang L, Felizola SJ, Ueno T, Toi M, Loo W, Chow LW, Suzuki T, Sasano H (2012) Changes of tumor infiltrating lymphocyte subtypes before and after neoadjuvant endocrine therapy in estrogen receptor-positive breast cancer patients-an immunohistochemical study of $\mathrm{Cd} 8+$ and FOXp3 + using double immunostaining with correlation to the pathobiological response of the patients. Int J Biol Markers 27(4):e295-e304

28. Bracci L, Schiavoni G, Sistigu A, Belardelli F (2014) Immunebased mechanisms of cytotoxic chemotherapy: implications for the design of novel and rationale-based combined treatments against cancer. Cell Death Differ 21(1):15-25

29. Andersen MH, Sorensen RB, Schrama D, Svane IM, Becker JC, Thor Straten P (2008) Cancer treatment: the combination of vaccination with other therapies. Cancer Immunol Immunother 57(11):1735-1743

30. Fareed KR, Ilyas M, Kaye PV, Soomro IN, Lobo DN, Parsons SL, Madhusudan S (2009) Tumour regression grade (TRG) analyses in patients with resectable gastro-oesophageal adenocarcinomas treated with platinum-based neoadjuvant chemotherapy. Histopathology 55(4):399-406

31. Ademuyiwa FO, Bshara W, Attwood K, Morrison C, Edge SB, Karpf AR, James SA, Ambrosone CB, O'Connor TL, Levine EG, Miliotto A, Ritter E, Ritter G, Gnjatic S, Odunsi K (2012) NY-ESO-1 cancer testis antigen demonstrates high immunogenicity in triple negative breast cancer. PLoS One 7(6):e38783

32. Loi SMS, Salgado R, Sirtaine N, Jose V, Fumagalli D, Kellokumpu-Lehtinen PL, Bono P, Kataja V, Desmedt C, Piccart MJ, Loibl S, Denkert C, Smyth MJ, Joensuu H, Sotiriou C (2014) Tumor infiltrating lymphocytes is prognostic and predictive for trastuzumab benefit in early breast cancer: results from the FinHER trial. Ann Oncol 25(8):1544-1550

33. Bang YJ, Van Cutsem E, Feyereislova A, Chung HC, Shen L, Sawaki A, Lordick F, Ohtsu A, Omuro Y, Satoh T, Aprile G, Kulikov E, Hill J, Lehle M, Ruschoff J, Kang YK, To GATI (2010) Trastuzumab in combination with chemotherapy versus chemotherapy alone for treatment of HER2-positive advanced gastric or gastro-oesophageal junction cancer (ToGA): a phase 3, open-label, randomised controlled trial. Lancet 376(9742):687-697
34. Tamura K, Shimizu C, Hojo T, Akashi-Tanaka S, Kinoshita T, Yonemori K, Kouno T, Katsumata N, Ando M, Aogi K, Koizumi F, Nishio K, Fujiwara Y (2011) FcgammaR2A and 3A polymorphisms predict clinical outcome of trastuzumab in both neoadjuvant and metastatic settings in patients with HER2-positive breast cancer. Ann Oncol 22(6):1302-1307

35. Lissoni P, Brivio F, Fumagalli L, Messina G, Ghezzi V, Frontini L, Giani L, Vaghi M, Ardizzoia A, Gardani GS (2004) Efficacy of cancer chemotherapy in relation to the pretreatment number of lymphocytes in patients with metastatic solid tumors. Int $\mathrm{J}$ Biol Markers 19(2):135-140

36. Gao Y, Zhou S, Jiang W, Huang M, Dai X (2003) Effects of ganopoly (a Ganoderma lucidum polysaccharide extract) on the immune functions in advanced-stage cancer patients. Immunol Invest 32(3):201-215

37. Chandler I, Houlston R, Landberg G (2011) A practical guide to constructing and using tissue microarrays. Methods Mol Biol 675:363-373

38. Altman DG, Royston P (2006) The cost of dichotomising continuous variables. BMJ 332(7549): 1080

39. Royston P, Altman DG, Sauerbrei W (2006) Dichotomizing continuous predictors in multiple regression: a bad idea. Stat Med 25(1):127-141

40. Ahmed SA, Talal N (1990) Sex hormones and the immune system Part 2 Animal data. Baillieres Clin Rheumatol 4(1):13-31

41. Ibrahim EM, Al-Foheidi ME, Al-Mansour MM, Kazkaz GA (2014) The prognostic value of tumor-infiltrating lymphocytes in triple-negative breast cancer: a meta-analysis. Breast Cancer Res Treat 148(3):467-476

42. Kim KJ, Lee KS, Cho HJ, Kim YH, Yang HK, Kim WH, Kang GH (2014) Prognostic implications of tumor-infiltrating FOXp3+ regulatory $\mathrm{T}$ cells and $\mathrm{CD} 8+$ cytotoxic $\mathrm{T}$ cells in microsatellite-unstable gastric cancers. Hum Pathol 45(2):285-293

43. Feichtenbeiner A, Haas M, Buttner M, Grabenbauer GG, Fietkau R, Distel LV (2014) Critical role of spatial interaction between $\mathrm{CD} 8(+)$ and FOXp3(+) cells in human gastric cancer: the distance matters. Cancer Immunol Immunother 63(2):111-119

44. Liu H, Zhang T, Ye J, Li H, Huang J, Li X, Wu B, Huang X, Hou J (2012) Tumor-infiltrating lymphocytes predict response to chemotherapy in patients with advance non-small cell lung cancer. Cancer Immunol Immunother 61(10):1849-1856 